\section{LEAKAGE DETERMINATION DURING REGIONAL PERFUSION}

BY

\author{
E. O. FIELD, D.M., D.M.R.D.
}

Radiobiological Research Unit, Institute of Cancer Research, Royal Cancer Hospital, Fulham Road, London

In the treatment of tumours by regional perfusion with chemotherapeutic agents it is important to assess the amount of the agent that escapes from the perfused site into the systemic circulation. Generally this is achieved by introducing a marker into the perfusion circuit and measuring the appearance of the marker on the systemic side. Radioactive tracers are especially suitable for this purpose since they enable the leakage rate to be assessed by external monitoring procedures.

In limb perfusions good surgical isolation of the perfused site can usually be achieved, and the value of monitoring is confined to ensuring that leakage does not arise during perfusion for accidental reasons, such as a misapplied tourniquet. However, when complete isolation cannot for technical reasons be achieved, monitoring is of especial value in that the leakage rate may be rapidly assessed with sufficient accuracy to indicate either how long perfusion may with safety be continued or, if the chemotherapeutic agent is administered in separate small fractions, the maximum dose that can safely be perfused.

The monitoring procedures employed in the perfusions described (p. 1231) by Garai, Cooling, and Staunton (1962) were based on those devised by Stehlin et al. (1960) and Stehlin, Clark, and Dewey (1961). The main differences in our method, however, lie in a simplification of apparatus and in the use of alternative criteria of leakage.

\section{Method}

A scintillation counter with wide-angle collimation was attached by a specially constructed adjustable clamp to the operating-table and directed horizontally across the table at the approximate level of the patient's heart in the mid-axillary line (Fig. 1). To reduce further the amount of equipment that might encumber the surgeon, the pre-amplifier and ratemeter were positioned in an adjoining room. The count rate was also registered visually by a pen recorder placed in the theatre within view of the surgeon.

A calibrating dose of radioisotope was injected systemically as soon as all tourniquets had been applied and a known multiple of the calibration dose introduced into the external pump reservoir. Approximately 10 minutes later, just before perfusion was started, a sample of systemic blood was obtained by venepuncture. A second sample of systemic blood and samples of blood from the arterial and venous limbs of the extracorporeal pump were taken either at the end of perfusion or at any intermediate stage, if a sudden change in the leakage rate was expected-for example, as a result of surgical intervention. In the latter event, further samples were obtained later as required.

Either $40 \mu \mathrm{c}$ of ${ }^{131}$ I-labelled human serum albumin (H.S.A.) or $100 \mu \mathrm{c}$ of ${ }^{51} \mathrm{Cr}$-labelled red cells were used, approximately one-fifth of the total being injected systemically for calibration. ${ }^{131}$ I H.S.A. was preferred as it could be used immediately, but owing to the short half-life of the isotope (8.1 days) this material was not always available. ${ }^{51} \mathrm{Cr}$, with its longer half-life $(27.8$

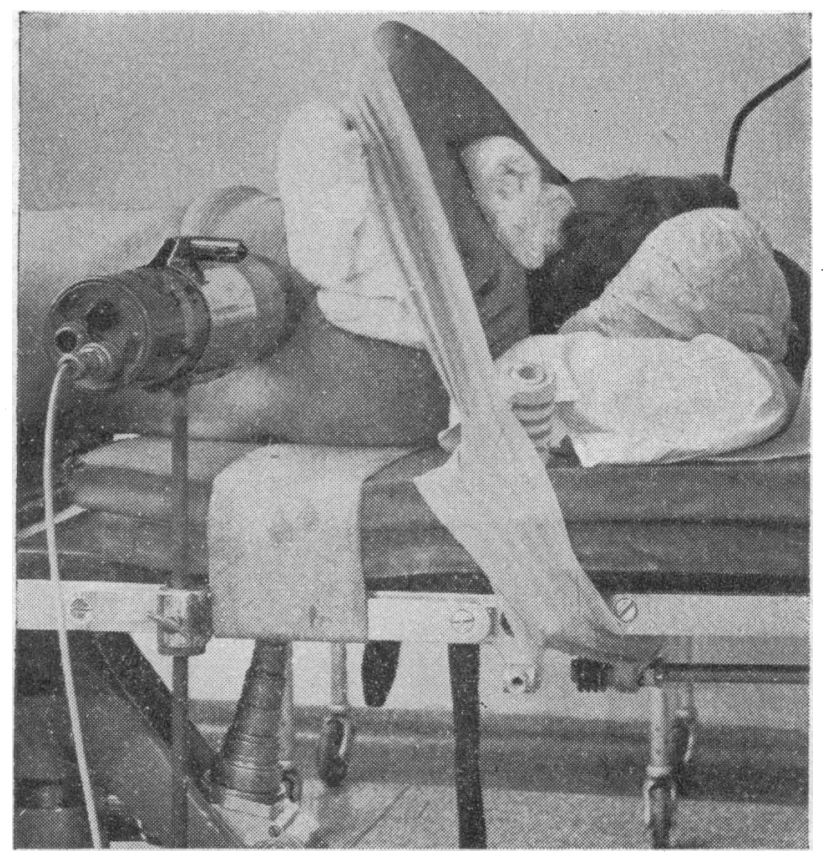

Fig. 1.-Arrangement for attaching scintillation counter to operating-table with adjustable clamp.

days), could be more easily stored, but was less convenient, since it necessitated labelling red cells before the operation.

\section{Calculations}

When leakage cannot be avoided estimates both of leakage rate and of total amount leaked may be required. The calculations are based on the assumptions, firstly, that the system may be represented by a closed two-compartment model in which the same volume of blood leaks out of one compartment (the perfused circulation) as in the reverse direction, in any interval of time; and, secondly, that the chemotherapeutic agent leaks only in one direction out of the perfused into the systemic compartment. The justifications for these assumptions are discussed later. It can be shown (Austen and Raker, 1960) that the distribution of isotope between the two compartments at any time is described by the equation

$$
k t=\frac{S}{S+P} \ln \left[\frac{q_{o}-\frac{D S}{S+P}}{q_{t}-\frac{D S}{S+P}}\right] .
$$

where $k$ =leakage rate from perfused to systemic circulation (fraction per unit time), $t=$ time perfused, $\mathbf{S}$ and $\mathbf{P}=$ systemic and perfused compartment volumes, $\mathrm{D}=$ total amount of isotope used, and $\mathrm{q}_{\mathrm{o}}$ and $\mathrm{q}_{\mathrm{t}}=$ amounts of isotope present in the systemic compartment initially and at time $t$.

The amounts of isotope in the two compartments at any time cannot be directly measured, but since the isotope content of a compartment is given by the 
product of compartment volume and isotope concentratlon (activity per unit volume), by putting

$\mathrm{x}=$ (concentration at time $t$ ) / (initial concentration) of isotope in compartment $S=q_{k} / q_{o}$,

$y=$ (concentration in compartment $S$ ) / (concentration in compartment $\mathrm{P}$ ) at time $t$, and

$\mathrm{z}=$ the fraction of the total dose used for calibration $=\mathrm{q}_{0} / \mathrm{D}$,

the above equation may be written in the form

$$
k t=\frac{S}{S+P} \ln \left[\frac{\frac{S}{S+P} \cdot \frac{1}{z}-1}{\frac{S}{S+P} \cdot \frac{1}{z}-x}\right]
$$

This is the general equation for deriving either leakage rate (k) or the total fraction leaked, which is equal to $1-\mathrm{e}^{-\mathrm{kt}}$.

If these estimates are made from the ratemeter recordings of the external monitoring system alone approximate values must be assumed for the relative compartment sizes in order to evaluate $\frac{S}{S+P}$. (These values may be based on previous experience gained with the sample-counting procedure.) If, however, the estimates are made from measured activities of blood samples, since it can be shown that

$$
\frac{S+P}{S}=1-y+y / x z
$$

no assumptions need be made concerning relative compartment volumes.

With either method the calculations of leakage can be facilitated by working with a fixed value of $z$, the fraction of the total dose of isotope used for calibration, say of 0.1. Graphs may then be prepared in advance, in which $k t$ and $1-e^{-k t}$ are plotted together along one axis against different values of $\mathrm{x}$, within the range likely to be required, plotted along the other. With these graphs leakage may be estimated rapidly during perfusion from the ratemeter recordings. If the samplecounting method is employed the leakage parameters may be equally rapidly derived from tables prepared for the required ranges of values of $x$ and $y$. Alternatively, if a predetermined value for $z$ cannot be used the solution of the general equation (above) may be speeded up by the use of a successive alignment type of nomogram.

Example.-Fig. 2 shows a typical ratemeter recording obtained during a pelvic perfusion. The data obtained by the external monitoring and by the sample-counting methods between 22.5 and 37.5 minutes, and the leakage parameters calculated from them, are listed in the Table. In this case the two methods gave identical results.

\section{Discussion}

It must be appreciated that the two-compartment model is a purely theoretical concept and that results calculated from equations based on this model provide only approximate estimates of actual leakage. Moreover, the leakage parameters provide measures only of the efficiency of isolation of a perfused region-not of the rate of escape of a drug into or of its accumulation in unperfused regions. The behaviour of a drug depends, in addition, on such biochemical and physiological properties as diffusibility into extravascular compartments, fixation in tissues, and rates of excretion or inactivation.

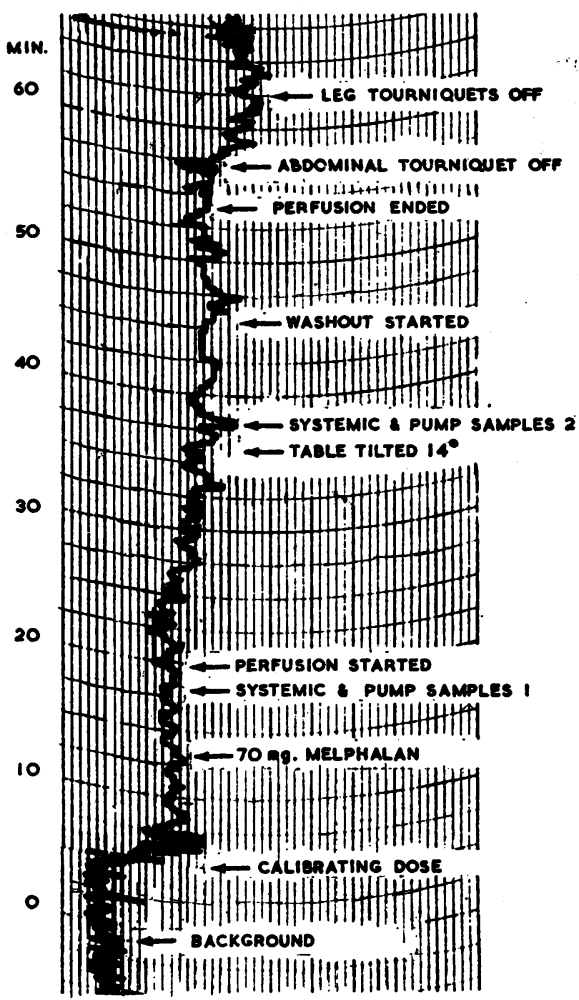
Fio. 2.-Example of a ratemeter tracing obtained during a pelvic
perfusion.

Comparison of Leakage Parameters Derived by External Monitoring and by Sample-counting Methods $(z=0.1 ; t=$ 15 min.)*

\begin{tabular}{c|c|c}
\hline & External Monitoring & Sample-Counting \\
\hline $\mathbf{x}$ & 1.67 & 1.76 \\
$\mathbf{S}$ & - & 0.172 \\
$\mathbf{S}+\mathbf{P}$ & Assumed 0.5 & 0.531 \\
Leakage rate, $\mathbf{k}(\%$ per min.) & 0.67 & 0.68 \\
Total leaked, \%, $\%\left(1-e^{-k t}\right) 100$ & 9.5 & 9.5 \\
\hline
\end{tabular}

- Symbols are defined in the text.

Stehlin et al. (1960) describe an alternative parameter, the leakage factor, defined as the radioactivity in the systemic circulation above calibration level, at any time, divided by the activity that would be present if intermixing were complete. We prefer, however, an estimate of leakage rate, since this is of greater value for those cases where the chemotherapeutic agent is administered in separate small fractions. Our parameter, the total fraction leaked, gives a measure of the maximum theoretical amount of drug that could possibly have accumulated in the unperfused regions, with the observed leakage rate. In most cases this parameter offers a margin of safety that is unnecessarily large, but it becomes of greater importance the smaller the relative size of the region perfused, especially with drugs that are rapidly fixed by tissues.

\section{Summary}

A procedure is described for estimating leakage during regional perfusion by means of radioactive tracers and external monitoring. Two parameters are definedleakage rate and total fraction leaked. The applicability of these parameters to the estimation of the amount of drug escaping from perfused to unperfused regions is discussed. An arrangement is illustrated for reducing 
the amount of equipment in the vicinity of the operating-table.

I am grateful to Professor D. W. Smithers, director of the department of radiotherapy, for encouraging me to develop this monitoring technique. My thanks are also due to Drs. K. W. Taylor and J. B. N. Stedeford, of the physics department, for their help in assembling the electronic equipment.
REFERENCES

Austen, W. G., and Raker, J. W. (1960). Cancer Chemother. Rep., 10, 61

Garai, O. F., Cooling, C. I., and Staunton, M. D. (1962). Brit. med. J., 1, 1231 .

Stehlin, J. S., Clark, R. L., White, E. C., Healey, J. E., Dewey, W. C., and Beerstecher, S. (1960). Arch. Surg., 80, 934.

\section{RECURRENT CANCER OF HEAD AND NECK \\ TREATMENT WITH CONTINUOUS INTRA-ARTERIAL METHOTREXATE AND INTERMITTENT INTRAMUSCULAR CITROVORUM FACTOR}

BY

\section{G. WESTBURY, F.R.C.S., M.R.C.P. Consultant Surgeon}

J. G. HUMBLE, C.V.O., M.R.C.P. Consultant Haematologist

D. E. PEGG,* M.B., B.S. Lecturer in Haematology

\author{
K. A. NEWTON, M.R.C.P., F.F.R. \\ Consultant Radiotherapist
}

H. T. FORD, M.B., B.S., F.F.R.

Senior Registrar, Radiotherapy Department

AND

W. F. WHITE, M.B., B.S.

Senior House Officer, Radiotherapy Department

Westminster Hospital, London

[With Special Plate]

Administration of cytotoxic agents directly into the artery of a tumour-bearing area was first described by Klopp and his colleagues (1950), who used fractionated doses of nitrogen mustard $\left(\mathrm{HN}_{2}\right)$. They showed that changes could be produced in normal and neoplastic tissues that were not achieved by intravenous injection even of lethal amounts of the drug. Spill-over into the general circulation did, however, produce moderate depression of haemopoiesis, and the authors suggested, among other methods, that systemic toxicity might be reduced by an antagonist given intravenously at the time of each intra-arterial injection. Sullivan et al. (1959), applying this principle, reported the use of continuous intra-arterial infusion of lethal doses of an antimetabolite together with the intermittent intramuscular administration of the specific metabolite as an antidote. They described remarkable regression of advanced tumours of the head and neck with intracarotid infusion of methotrexate (4-amino- $\mathrm{N}^{10}$-methylpteroyl glutamic acid; amethopterin), a folic-acid analogue which interferes with the participation of folic acid in nucleic-acid synthesis ; citrovorum factor (folinic acid ; leucovorin) was given in amounts sufficient to minimize systemic toxicity, but not to compete entirely with the high concentrations of antimetabolite in the carotid territory.

The present paper relates our experience with this method of therapy in a group of patients with inoperable malignant tumours of the head and neck, all of whom had been heavily treated with one or more courses of supervoltage radiotherapy. It was anticipated that delayed irradiation effects, in particular endarteritis, might reduce the efficacy of intra-arterial therapy, but it was not thought justifiable at this stage to use a new technique of treatment before giving patients the benefit of radiotherapy and/or surgery, methods known to be capable of achieving control of disease for many years. This problem is further considered in the discussion.

\footnotetext{
*In receipt of a full-time grant from the British Empire Cancer Campaign during the period of this study.
}

\section{Material}

Since July, 1960, 26 cases have been treated. Two of these received a second infusion after 9 and 11 months respectively. The lesions treated included 3 carcinomas of tongue, 1 carcinoma of lip, 11 carcinomas of buccal mucosa and alveolus, 4 carcinomas of palate, antrum, or ethmoid, 2 carcinomas of tonsillar fossa, 2 primary skin carcinomas, 2 rhabdomyosarcomas of mouth and orbit respectively, and 1 fibrosarcoma of parotid region. The youngest patient was aged 7 and the oldest 87 years. All patients, as stated, had received supervoltage radiotherapy in radical dosage. Seven cases had been treated twice, one three times, and one at least five times over some years. In 12 cases local or radical excision of the primary lesion had been performed; two patients had undergone multiple surgical procedures. Four patients had had block dissection of cervical lymph nodes. Case 13 is a typical example. This 66-year-old man with carcinoma of the buccal mucosa was treated by implantation of radium needles in September, 1959, to a dose of $8,000 \mathrm{r}$ in seven days. A local recurrence was treated in March, 1960, with the 15-curie telecobalt unit to 5,592 $\mathrm{r}$ in 41 days, and in June, 1960, hemimandibulectomy and block dissection of the neck in continuity was performed. Further local recurrence failed to respond to an implant of gold grains in November, 1960, and the patient was presented for infusion therapy in March, 1961.

Details of the 26 cases are given in the Table.

\section{Method}

In most cases the tumour lay within the territory of supply of the external carotid artery, which was usually cannulated via its superior thyroid branch (Klopp et al.) exposed through a short incision at the level of the hyoid bone. Polyvinyl chloride ("portex") tubing was employed, being easier to secure than polyethylene, as it $i$ soft enough to take the bite of the encircling ligatures; a modified No. 6 F.G. umbilical 